\title{
Elizabethkingia anophelis Infection in Infants, Cambodia, 2012-2018
}

\author{
Thomas A.N. Reed, Gabriella Watson, Chheng Kheng, Pisey Tan, \\ Tamalee Roberts, Clare L. Ling, Thyl Miliya, Paul Turner
}

We describe 6 clinical isolates of Elizabethkingia anophelis from a pediatric referral hospital in Cambodia, along with 1 isolate reported from Thailand. Improving diagnostic microbiological methods in resource-limited settings will increase the frequency of reporting for this pathogen. Consensus on therapeutic options is needed, especially for resource-limited settings.

Flizabethkingia anophelis is a recently identified aerEobic, nonmotile, oxidase-positive, indole-positive species of gram-negative bacillus $(1,2)$ that has been implicated in nosocomial and community outbreaks and associated with high mortality rates $(3,4)$. We report a case of $E$. anophelis bacteremia in an infant in Cambodia in October 2018 and a retrospective study to identify previously misidentified isolates and describe the clinical features of E. anophelis-associated pediatric illness in Cambodia.

\section{The Study}

A 7-day-old girl was brought to her local hospital with difficulty in breathing and poor feeding. She was a twin, born vaginally at 36 weeks' gestation, with no antenatal or delivery complications. Both her mother and twin were in good health. Hospital staff administered ampicillin $(50 \mathrm{mg} / \mathrm{kg} 2 \times / \mathrm{d})$ and gentamicin $(5 \mathrm{mg} / \mathrm{kg} / 36 \mathrm{~h})$.

The patient was transferred to the pediatric intensive care unit of Angkor Hospital for Children, a nongovernmental pediatric hospital in Siem Reap, Cambodia, with presumed late-onset neonatal sepsis. Upon arrival, she was cyanotic with recurrent apneas, requiring intubation, and had jaundice. Clinical examination and vital signs were otherwise unremarkable. Blood tests showed a leukocyte count of $8.5 \times 10^{9} / \mathrm{L}$ (neutrophils $6.4 \times 10^{9} / \mathrm{L}$ ); hemoglobin $16.9 \mathrm{~g} / \mathrm{dL}$; platelet count

Author affiliations: Angkor Hospital for Children, Siem Reap, Cambodia (T.A.N. Reed, G. Watson, C. Kheng, P. Tan, T. Miliya, P. Turner); Lao-Oxford-Mahosot Hospital, Vientiane, Laos

(T. Roberts); Mahidol University, Mae Sot, Thailand (C.L. Ling); University of Oxford, Oxford, UK (C.L. Ling, P. Turner)

DOI: https://doi.org/10.3201/eid2602.190345
$45 \times 10^{9} / \mathrm{L}$; C-reactive protein $195 \mathrm{mg} / \mathrm{L}$; and total bilirubin $252 \mu \mathrm{mol} / \mathrm{L}$. Lumbar puncture was omitted due to thrombocytopenia. A blood culture was transferred with her from the local hospital.

The day after transfer, she experienced symptoms of meningitis, including fever and seizures. We initiated anticonvulsant therapy and changed her antimicrobial therapy to intravenous meropenem $(40 \mathrm{mg} / \mathrm{kg} \mathrm{3 \times /d}$ ). Blood culture microscopy subsequently showed gram-negative bacilli, identified as E. anophelis on hospitalization day 3 by matrixassisted laser desorption/ionization time-of-flight (MALDI-TOF) mass spectrometry using bioMérieux VITEK MS in an in vitro diagnostic mode using the spectrum knowledge base version 3.2.0 (bioMérieux, https://www.biomerieux.com). At this stage antimicrobial drugs were changed to intravenous ciprofloxacin $(10 \mathrm{mg} / \mathrm{kg}, 2 \times / \mathrm{d})$ and vancomycin (15 $\mathrm{mg} / \mathrm{kg}, 1 \times / \mathrm{d})$; a blood culture collected before the change confirmed bacteremia caused by E. anophelis.

The patient was extubated on day 6 and underwent lumbar puncture because her platelet count had improved. Cerebrospinal fluid was cloudy, with a leukocyte count of 265 cells/ $\mu \mathrm{L}$ (75\% polymorphs), glucose of $1 \mathrm{mmol} / \mathrm{L}$, and protein of $13 \mathrm{~g} / \mathrm{L}$. Gram stain microscopy revealed no organisms, and culture was negative. After 28 days of ciprofloxacin/vancomycin, she was clinically well and discharged home.

At her 1-month follow-up appointment, she displayed clinical features of raised intracranial pressure, including neurologic deficits. Cranial ultrasound showed hydrocephalus, a suspected sequela of meningitis, and she was referred for neurosurgical opinion.

After the case described was identified, we retrieved all isolates in $-80^{\circ} \mathrm{C}$ storage that had been identified since January 2012 as Chryseobacterium meningosepticum, C. miricola, or Elizabethkingia spp. We included in our study the first isolates from a given clinical episode: 4 identified as C. meningosepticum, 3 as E. meningoseptica, and the isolate already identified as E. anophelis. From subculture, we analyzed these using VITEK MS MALDI-TOF mass spectrometry. We identified 
6 isolates as E. anophelis and 1 as E. meningoseptica. MALDI-TOF was unable to return an identification for 1 isolate, previously identified as C. meningosepticum.

We determined MICs to antimicrobial drugs for all E. anophelis isolates using Etest (bioMérieux). $\mathrm{MIC}_{50}$ result for ceftriaxone was $64 \mu \mathrm{g} / \mathrm{mL}$; for sulfamethoxazole/trimethoprim, $0.25 \mu \mathrm{g} / \mathrm{mL}$; for ciprofloxacin, 0.5 $\mu \mathrm{g} / \mathrm{mL}$; and for vancomycin, $12 \mu \mathrm{g} / \mathrm{mL}$ (Table).

To provide regional context for these results, 2 microbiology laboratories in Mae Sot, Thailand, and Vientiane, Laos, also reanalyzed stored clinical isolates as we described. In Mae Sot, a single isolate of E. meningoseptica from a neonatal blood culture was reidentified as E. anophelis. In Vientiane, 9 isolates of C. meningosepticum were reidentified as E. meningoseptica, and the identity of 1 E. meningoseptica isolate remained the same.

\section{Conclusions}

Although reports of E. anophelis are rare, cases are reported from countries in southern Asia, including Singapore (3), Taiwan (5), and Hong Kong (6). Our findings are consistent with reports of $E$. anophelis infection from other countries demonstrating it to be an opportunistic organism affecting more vulnerable patient groups (6). The mortality rate associated with
E. anophelis is high (50\%), and isolation of E. anophelis from blood in two thirds of the children in this study demonstrates its importance as a human pathogen.

Previous reports of community- and hospital-acquired E. anophelis infection among infants have proposed a range of transmission routes, including vectorborne (Anopheles mosquitoes) $(1,2,7)$, waterborne (8), and vertical transmission (9). With no temporal clustering, and with most cases occurring among older infants, we suspect that unidentified environmental reservoirs are possible sources of these cases.

Previously, studies relied on 16S rRNA testing to identify E. anophelis, with biochemical phenotypic methods unable to distinguish between Elizabethkingia spp. (10). Although this method provides high discriminatory power, its use in diagnostic microbiology is limited to established laboratory settings. It also requires highly trained staff to interpret results, which are rarely available within a clinically useful timeframe. Until late 2017, oxidase-positive gram-negative isolates were identified at the microbiology laboratory at Angkor Hospital for Children by biochemical phenotypic methods (API 20NE, bioMérieux); identification is now done by MALDI-TOF mass spectrometry. Misidentification of Elizabethkingia spp. using biochemical methods has been reported $(2,6)$; however, updated MALDI-TOF databases

\begin{tabular}{|c|c|c|c|c|c|c|c|}
\hline \multirow[b]{2}{*}{ Characteristic } & \multicolumn{7}{|c|}{ Isolate no. } \\
\hline & 1 (this study) & 2 & 3 & 4 & 5 & 6 & 7 \\
\hline \multicolumn{8}{|l|}{ Patient characteristics } \\
\hline Sex & $\mathrm{F}$ & M & $\mathrm{F}$ & $M$ & $\mathrm{~F}$ & M & $\mathrm{F}$ \\
\hline Age at admission & $6 \mathrm{~d}$ & $8 \mathrm{mo}$ & $15 \mathrm{wk}$ & $0 \mathrm{~d}$ & $51 \mathrm{~d}$ & $0 \mathrm{~d}$ & $0 \mathrm{~d}$ \\
\hline Concurrent condition & Prematurity† & $\begin{array}{l}\text { Duodenal } \\
\text { atresia }\end{array}$ & $\begin{array}{l}\text { Failure to } \\
\text { thrive }\end{array}$ & Prematurity $†$ & $\begin{array}{l}\text { Ventricular } \\
\text { septal defect }\end{array}$ & Prematurity† & Prematurity† \\
\hline Country & Cambodia & Cambodia & Cambodia & Cambodia & Cambodia & Cambodia & Thailand \\
\hline \multicolumn{8}{|l|}{ Clinical features } \\
\hline Diagnosis & Meningitis & VAP & Meningitis & Sepsis & VAP & Sepsis & Sepsis \\
\hline Treatment & CIP/NAN & MER & CAX & AMP/GM & CIP & IMP & AMP/GM \\
\hline Outcome & Survived & Died & Unknownł & Died & Died & Survived & Died \\
\hline Length of admission, $d$ & 31 & 16 & 1 & 5 & 79 & 35 & 25 \\
\hline \multicolumn{8}{|l|}{ Specimen details } \\
\hline Collection date & 2018 Oct & 2018 Jan & 2015 Aug & 2013 Aug & 2012 Sep & 2012 Mar & $2017 \mathrm{Apr}$ \\
\hline Specimen type & Blood & $\begin{array}{l}\text { Respiratory } \\
\text { secretion }\end{array}$ & Blood & Blood & $\begin{array}{l}\text { Respiratory } \\
\text { secretion }\end{array}$ & Blood & Blood \\
\hline $\begin{array}{l}\text { Hospitalization day } \\
\text { collected }\end{array}$ & 1 & 16 & 1 & 5 & 64 & 21 & 22 \\
\hline \multicolumn{8}{|l|}{ Isolate details } \\
\hline First ID & E. anophelis & \multicolumn{2}{|c|}{ E. meningoseptica } & \multicolumn{3}{|c|}{ C. meningosepticum } & $E$. \\
\hline Initial ID method & MALDI-TOF & MALDI-TOF & API 20NE & API 20NE & API 20NE & API 20NE & $\begin{array}{c}\text { meningoseptica } \\
\text { API 20NE }\end{array}$ \\
\hline \multicolumn{8}{|l|}{$\mathrm{MIC}(\mu \mathrm{g} / \mathrm{mL})$} \\
\hline VAN & 8 & 16 & 16 & 16 & 8 & 16 & 8 \\
\hline SXT & 0.25 & 0.25 & 0.25 & 0.5 & 0.25 & 0.5 & NA§ \\
\hline CAX & 32 & 64 & $>256$ & 64 & 64 & $>256$ & $>256$ \\
\hline CIP & 1 & 0.5 & 0.5 & 0.5 & 0.5 & 0.5 & 1 \\
\hline
\end{tabular}


provide reliable differentiation (10). As the resolution that MALDI-TOF mass spectrometry provides in pathogen identification expands, and its use becomes available in low- and middle-income countries, we expect to see higher reported incidence of $E$. anophelis infection. Conversely, it may become apparent that the burden of $E$. meningoseptica is not as high as previously thought, with retrospective studies already showing $E$. anophelis as the predominant species of its genus $(6,10,11)$. In our study, this possibility was not found to be the case in Laos, suggesting possible regional variation.

E. anophelis demonstrates phenotypic and genotypic resistance to multiple antimicrobial drugs, and, without epidemiologically based interpretive cutoffs, selection of therapeutic options is challenging $(4,5,10,12)$. High MICs to ceftriaxone are consistent with $\beta$-lactam resistance reported elsewhere, and carbapenem resistance should also be expected $(4,5,10,12)$. Following Clinical and Laboratory Standards Institute guidelines (M100-29; 2019) (13) for "other non-Enterobacteriaceae," these isolates were susceptible to ciprofloxacin and sulfamethoxazole/trimethoprim. This finding is not consistent with other regional data that show greater rates of resistance to these drugs $(5,10)$. E. anophelis has been shown to be susceptible to piperacillin/tazobactam and to rifampin $(4,10)$, which were not tested against in this study and are not currently available as therapeutic options in the study setting. It is unusual for gram-negative organisms to exhibit susceptibility to vancomycin, and interpretation of MICs to this drug should be approached with caution. Use of Etest in this study was a methodological limitation; the preferred method of broth microdilution was not available.

In summary, updates of mass spectrometry platforms have enabled identification of clinical E. anophelis isolates in Cambodia and Thailand. As diagnostic microbiology capacity expands in low- and middleincome countries, further reports of this organism are expected. Because of the associated high mortality rates for this pathogen, consensus on therapeutic options for infection caused by E. anophelis is needed, especially in resource-limited settings with restricted choices for antimicrobial drugs.

\section{Acknowledgments}

We thank Verena Carrara and the microbiology department of Shoklo Malaria Research Unit for their assistance in laboratory work and collecting of clinical details.

\section{About the Author}

Dr. Reed is a research clinician at the Cambodia-Oxford Medical Research Unit, Angkor Hospital for Children, Siem Reap, Cambodia. His primary research interest is antimicrobial resistance.

\section{References}

1. Kämpfer P, Matthews H, Glaeser SP, Martin K, Lodders N, Faye I. Elizabethkingia anophelis sp. nov., isolated from the midgut of the mosquito Anopheles gambiae. Int J Syst Evol Microbiol. 2011;61:2670-5. https://doi.org/10.1099/ ijs.0.026393-0

2. Frank T, Gody JC, Nguyen LBL, Berthet N, Le FlecheMateos A, Bata P, et al. First case of Elizabethkingia anophelis meningitis in the Central African Republic. Lancet. 2013;381:1876. https://doi.org/10.1016/S0140-6736 (13)60318-9

3. Teo J, Tan SY, Tay M, Ding Y, Kjelleberg S, Givskov M, et al. First case of $E$ anophelis outbreak in an intensive-care unit. Lancet. 2013;382:855-6. https://doi.org/10.1016/ S0140-6736(13)61858-9

4. Perrin A, Larsonneur E, Nicholson AC, Edwards DJ, Gundlach KM, Whitney AM, et al. Evolutionary dynamics and genomic features of the Elizabethkingia anophelis 2015 to 2016 Wisconsin outbreak strain. Nat Commun. 2017;8:15483. https:// doi.org/10.1038/ncomms15483

5. Lin JN, Lai CH, Yang $\mathrm{CH}$, Huang YH. Comparison of clinical manifestations, antimicrobial susceptibility patterns, and mutations of fluoroquinolone target genes between Elizabethkingia meningoseptica and Elizabethkingia anophelis isolated in Taiwan. J Clin Med. 2018;7:538. https:/ / doi.org/ 10.3390/jcm7120538

6. Lau SK, Chow WN, Foo CH, Curreem SO, Lo GC, Teng JL, et al. Elizabethkingia anophelis bacteremia is associated with clinically significant infections and high mortality. Sci Rep. 2016;6:26045. https://doi.org/10.1038/srep26045

7. Raygoza Garay JA, Hughes GL, Koundal V, Rasgon JL, Mwangi MM. Genome sequence of Elizabethkingia anophelis strain EaAs1, isolated from the Asian malaria mosquito Anopheles stephensi. Genome Announc. 2016;4:e00084-16. https://doi.org/10.1128/genomeA.00084-16

8. Yung CF, Maiwald M, Loo LH, Soong HY, Tan CB, Lim PK, et al. Elizabethkingia anophelis and association with tap water and handwashing, Singapore. Emerg Infect Dis. 2018;24:1730-3. https:// doi.org/10.3201/eid2409.171843

9. Lau SK, Wu AK, Teng JL, Tse H, Curreem SO, Tsui SK, et al. Evidence for Elizabethkingia anophelis transmission from mother to infant, Hong Kong. Emerg Infect Dis. 2015;21:23241. https:// doi.org/10.3201/eid2102.140623

10. Han MS, Kim H, Lee Y, Kim M, Ku NS, Choi JY, et al. Relative prevalence and antimicrobial susceptibility of clinical isolates of Elizabethkingia species based on $16 \mathrm{~S}$ rRNA gene sequencing. J Clin Microbiol. 2017;55:274-80. https://doi.org/10.1128/JCM.01637-16

11. Chew KL, Cheng B, Lin RTP, Teo JWP. Elizabethkingia anophelis is the dominant Elizabethkingia species found in blood cultures in Singapore. J Clin Microbiol. 2018;56:e0144517. https:// doi.org/10.1128/JCM.01445-17

12. Breurec S, Criscuolo A, Diancourt L, Rendueles O, Vandenbogaert M, Passet V, et al. Genomic epidemiology and global diversity of the emerging bacterial pathogen Elizabethkingia anophelis. Sci Rep. 2016;6:30379. https://doi.org/10.1038/srep30379

13. Clinical and Laboratory Standards Institute (CLSI). Performance standards for antimicrobial susceptibility testing; 29th edition (M100-29). Wayne (PA): The Institute; 2019.

Address for correspondence: Thomas A.N. Reed, Cambodia-

Oxford Medical Research Unit, Angkor Hospital for Children, Tep Vong (Achamean) Rd and Oum Chhay St, Svay Dangkum, PO Box 50, Siem Reap, Cambodia; email: tanreed@gmail.com 